\title{
Toward a Typology of Displacements in the Context of Slow-Onset Environmental Degradation. An Analysis of Hazards, Policies, and Mobility Patterns
}

\author{
Raoul Kaenzig and Etienne Piguet *(D) \\ Institute of Geography, University of Neuchâtel, 2000 Neuchâtel, Switzerland; Raoul.Kaenzig@unine.ch \\ * Correspondence: Etienne.Piguet@unine.ch
}

Citation: Kaenzig, R.; Piguet, E. Toward a Typology of Displacements in the Context of Slow-Onset Environmental Degradation. An Analysis of Hazards, Policies, and Mobility Patterns. Sustainability 2021, 13, 10235. https://doi.org/10.3390/ su131810235

Academic Editor: Donato Morea

Received: 12 August 2021

Accepted: 9 September 2021

Published: 14 September 2021

Publisher's Note: MDPI stays neutral with regard to jurisdictional claims in published maps and institutional affiliations.

Copyright: (c) 2021 by the authors. Licensee MDPI, Basel, Switzerland. This article is an open access article distributed under the terms and conditions of the Creative Commons Attribution (CC BY) license (https:/ / creativecommons.org/licenses/by/ $4.0 /)$.

\begin{abstract}
The aim of this paper is to develop a typology of displacement in the context of slow-onset environmental degradation linked to climate change (desertification, droughts and increasing temperatures, sea level rise (SLR), loss of biodiversity, land/forest degradation, and glacial retreat). We differentiate regions under environmental threat according to their social vulnerabilities, mobility patterns, and related policies, and identify twelve types of vulnerability/policy/mobility combinations. The paper is based on a synthesis of 321 published case studies on displacement and slow-onset environmental degradation, representing a comprehensive collection of the literature since the 1970s. We observe that vulnerability is especially critical in small island and coastal contexts, as well as in mountainous zones and desert regions. Migration processes are often not visible in areas affected by environmental degradation. When they do occur, they remain mostly internal and oriented towards cities with occasional rural-to-rural migration. Non-mobile people, as well as those who depend on natural resource industries for their livelihoods, are particularly vulnerable to climate change. Persons with lower levels of education are more likely to respond to environmental shock through short-distance migration, whereas highly educated individuals may migrate over longer distances. Policies that directly address mobility in relation to climate change-mostly through relocation-are seldom mentioned in the literature. Mobility is often perceived as a last-resort solution, whereas a growing body of research identifies mobility as an adaptation strategy.
\end{abstract}

Keywords: migration; mobility; climate; environmental change

\section{Introduction}

The literature that assesses the impact of environmental hazards, climate change, and climate variability on human displacement and migration often distinguishes between slow- and fast-onset events [1]. Droughts, sea level rise, coastal erosion, glacial melting and the degradation associated with such processes are usually labeled "slow-onset", whereas hurricanes, flash floods, torrential rains, landslides, earthquakes, and tsunamis are labeled "fast-onset" or "sudden onset". A second distinction is made between direct and indirect links. For example, a direct link occurs if coastal erosion forces villagers to relocate or if a hurricane causes people to flee. An indirect link occurs if progressive desertification affects traditional farming practices, leading some people to leave the affected region, or if a drought exacerbates conflicts over resources, contributing to violence that may push people to flee [2]. In this regard, mobilities related to slow-onset are more often indirect, whereas the consequences of sudden disaster are tendentially more straightforward.

In practice, there is often a continuum between "fast and slow onset" as well as between "direct and indirect impact", making clear-cut distinctions impossible. Drought, for example, can be considered fast or slow according to circumstances. Furthermore, the degree to which a given society or community is (un)able to adapt to changing environmental conditions might completely modify the final impact. In any case, none of these links are considered "natural". Human (re-)actions alter natural and environmental phenomena, 
and the same onset-whether fast or slow-will be experienced in different ways according to the social, religious, cultural, and economic context. In this exploratory study, we aim to build a typology of displacement contexts associated with slow-onset event profiles that helps clarify the various interactions between environmental hazards, societies, and mobility. We chose to focus on slow-onsets processes, although many selected studies also address fast-onset events.

\section{Materials and Methods}

This chapter explains how the typology, namely the list of the profiles, was constituted. We describe how we used the CliMig Bibliographical Database, define the parameters used to create the profiles, and discuss the advantages and limits of the study.

\subsection{Database}

The CliMig Database (https: / / climig.com, accessed on 24 September 2019) is a comprehensive collection of resources specific to displacement and migration, the environment, and climate change. It contains more than 1400 scientific articles dedicated to the environmental migration nexus (we followed IPCC guidelines in the selection of the literature: https://www.ipcc.ch/site/assets/uploads/2018/02/FS,_ipcc_assess.pdf, accessed on 24 September 2019). Following a standard procedure, each publication is assessed by experts and assigned a series of keywords to ensure scientific accuracy, consistency, and clarity. This systematic coding allows us to identify the region (country, continent, or other type of region (low elevation coastal zones, mountains, arctic zones, urban areas, etc.), the kind of environmental degradation (desertification, sea level rise, flood, etc.), the specific focus of the study (gender issues, juridical aspects, subjectivities, etc.) and the methodology (ethnographic fieldwork, longitudinal studies, etc.).

For this exploratory study, we focused the analysis on articles identified as case studies (a case study is here defined as empirical research that makes use of primary-source data for which the methodology of analysis can be identified (excluding, for example, syntheses, state of the art, policy briefs, or strictly descriptive collections of data, etc.) (based on Piguet 2010) with an emphasis on slow-onset environmental degradation (excluding hurricanes and floods), published up to June 2019. A set of 321 articles has therefore constituted the bibliographic basis for this study. The complete list of these articles is provided in the Supplementary Materials.

\subsection{Parameters}

Our point of departure considers existing case studies as accurate samples of the processes linking mobility and environmental degradation worldwide, keeping in mind that biases remain regarding the geography of research [3]. Further steps include implementing successive parameters in order to structure the most relevant typology and synthesizing the commonalities and differences within each identified profile.

We defined four parameters to classify the numerous references and build the typology: physical processes, geographical contexts, policies and mobility patterns. Each identified profile is the result of a combination of these parameters (see Figure 1). The choice for each of these parameters is subsequently explained. 
Parameters 1: Physical processes

Parameters 2:

Parameters 2:

Parameters 3:

Policies

\section{Parameters 4:}

Mobility patterns

Profiles
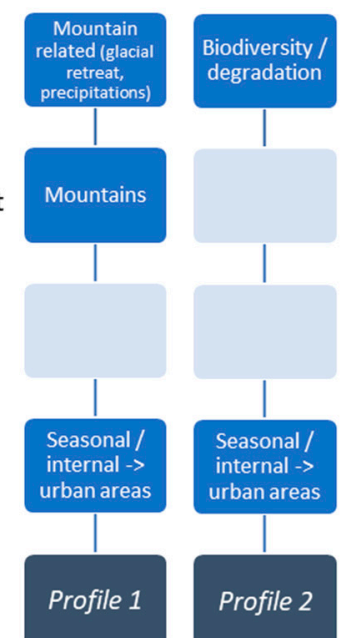
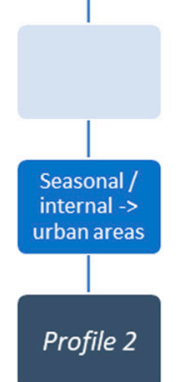

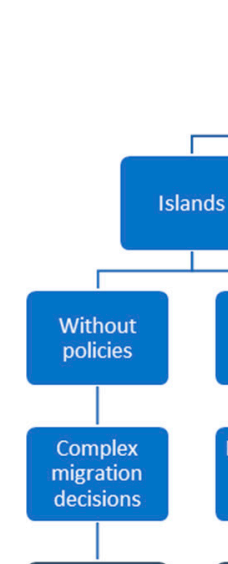

Profile $3 a$
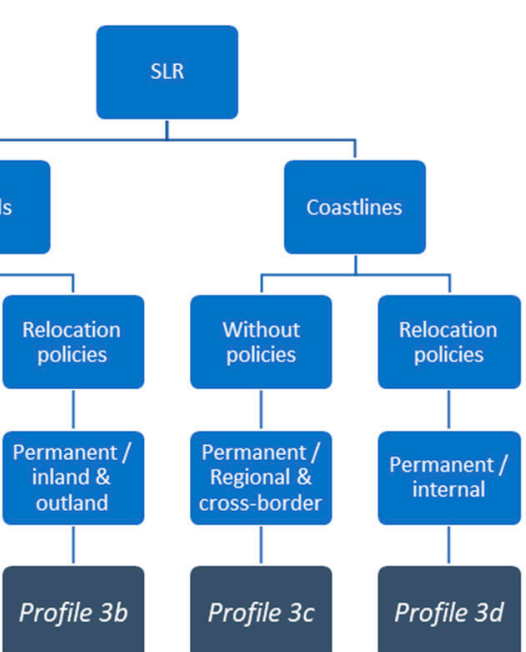
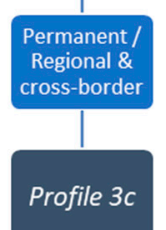
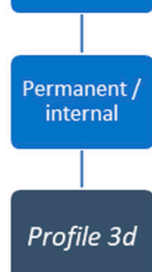

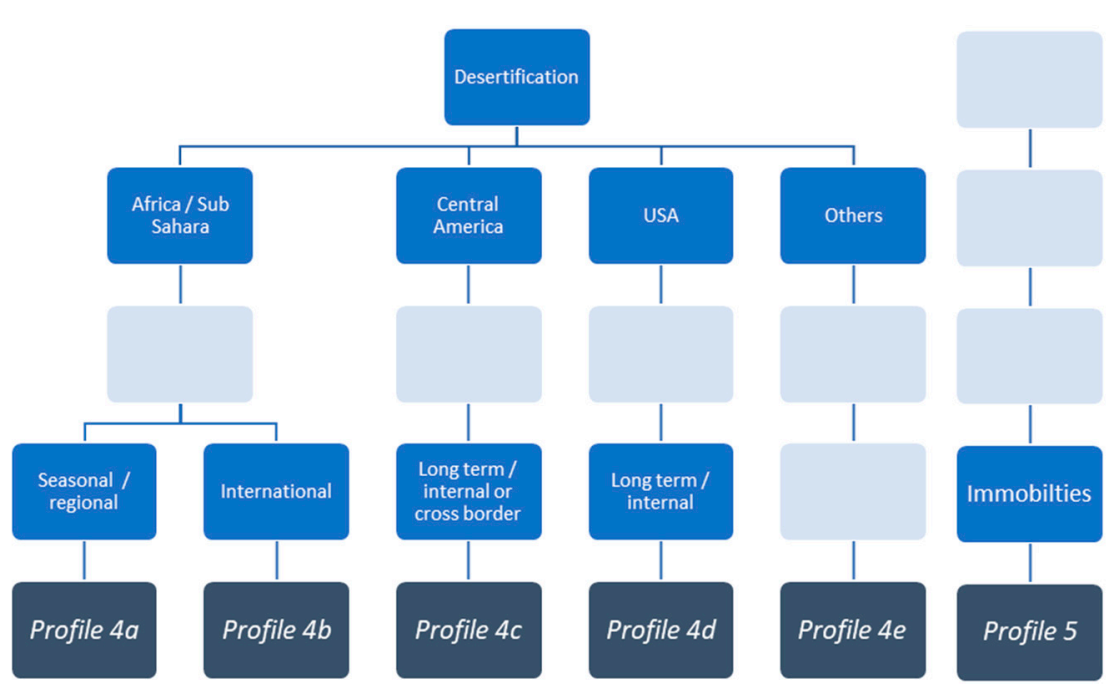

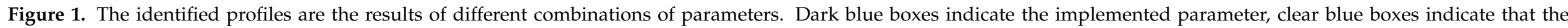
corresponding parameter has not been considered (i.e., not enough information or not relevant) for the selection of the profile. 


\subsubsection{Physical Processes}

The slow-onset event selection is based upon the thematic IDMC paper "No matter of choice: Displacement in a changing climate" (2018) [4], which refers to the typology developed within the UN Framework Convention on Climate Change (UNFCCC) classification. In this context, we consider case studies that address issues related to the following processes, keeping in mind that sudden degradations and disasters are often intermingled and taken into account in many studies:

- Desertification (including droughts and increasing temperatures), episodes of drought are sometimes considered extreme events, but since most studies do not differentiate them clearly from desertification, we decided to include them in this study;

- Sea level rise (SLR), ocean acidification is mentioned in the UNFCC classification, but we did not find any empirical articles documenting this phenomenon in the migration and environment literature;

- Loss of biodiversity and land/forest degradation;

- Glacial retreat.

\subsubsection{Geographical Context}

We considered two approaches to specifying geographical context. First, we identified ecoregions undergoing processes of glacial retreat or SLR (i.e., mountains, islands, coastlines). The affected locations are spread across the world; ecoregions appear to be relevant at this scale. Second, we specified the geographical context at the continental or sub-continental level for desertification (i.e., Africa, sub-Sahara, Central America, USA) because the ecoregional criteria tend to be similar (arid areas). Finally, the number of available case studies on biodiversity loss and land/forest degradation remains insufficient to consider geographical parameters.

\subsubsection{Migration-Related Policies}

Policies are understood here as national, regional, and global migration management policies and strategies (i.e., reinstallation/relocation programs or specific migration laws). However, the case studies reviewed show that policies are not a central topic in the relevant literature, which probably reflects the fact that they are often not actually implemented. In general, policy issues are more prevalent in articles with broader perspectives, such as syntheses, regional overviews, or juridical-oriented papers. An exception is to be found in papers focusing on SLR. Due to the non-reversible characteristics of this process, which may lead to inhabitability, relocation policies are often at the heart of these specific case studies. It can also be noted that the overlap between broader sets of policies not necessarily targeting mobility specifically (adaptation to climate change, humanitarian assistance, settlement policies for herders) often remain uncovered by the reviewed case studies.

\subsubsection{Mobility Patterns}

This parameter corresponds to the major characteristics of possible human mobility patterns:

- Distance (local, regional, international, etc.);

- Temporality (temporary, permanent, circular, etc.);

- Motivation (voluntary, forced, multifactorial, etc.);

- Migrants' characteristics (gender, race, age, education, etc.);

- Other specific situations (statelessness, non-migration, trapped population, etc.).

\subsubsection{Excluded Parameters}

Other parameters, such as socioeconomic factors, could have been considered in order to build our typology. However, these aspects are much less systematically discussed in the empirical literature-on which this paper is based—than geographical contexts, mobility 
patterns, and policy issues. As a result, this parameter will only be incidentally mentioned when migrant profiles are described.

\subsection{Challenges and Limits of Typologies}

In this exploratory study we aim to build a typology of displacement contexts associated with slow-onset event profiles. As previously described, the identification of profiles is based on a systematic analysis of the database using the aforementioned parameters. This work adds value to the current state of knowledge by creating an accessible and global understanding of how slow-onset hazards are related to human mobility. Due to their prolonged durations and interconnections with various migratory drivers, the nexus between migration and slow-onset environmental drivers is often characterized by complex and indirect correlations that are clarified through the identification of each profile.

This approach involves some methodological constraints and limits. The first issue is the order-or hierarchy-of implementing selected parameters. Considering the literature, we decided that physical processes should be the first parameter in the identification of profiles, followed by geographical context (ecoregions, continents, sub-continents), and the presence or absence of policies related to mobilities. This hierarchy emerges from the frequency with which each parameter appeared in case studies on environment and migration (which use different approaches, tackle various issues, and are based on distinctive scales of analysis). In this context, types of environmental degradation and the geographical characteristics of the studied area are clearly described in most studies, whereas mobility patterns and policies are not systematically assessed. For example, studies that address environmental factors in migration decisions rarely provide a comprehensive analysis of the mobility capabilities of migrants, or the policies that may support or inhibit their mobility.

The second issue relates to the wider question of the boundaries of each profile. Different contexts can be grouped within one profile because we found that a certain degree of homogeneity exists with regard to the environment, mobility, and/or policies. This homogeneity cannot be calculated using objective indicators. Therefore, the inclusion or exclusion of certain cases into a specific profile greatly depends on the author's evaluation of degree. Different parameters - or a different appreciation of them-will undoubtedly lead to another set of profiles. In this initial research, the procedure for profile identification was carried out as transparently as possible in order to allow for the deconstruction and/or reconstruction of categories

A third issue is that, although our typology intends to describe the various combinations of geographical contexts, mobilities, and policies, it is built on literature with pre-existing biases.

\section{Results: Typology of Displacement}

This chapter includes a list of identified profiles of displacement contexts associated with slow-onset events based on the parameters mentioned above. Each of the twelve distinctly identified profiles is numbered; those referring to similar hazards are also assigned a letter $(3 a, 3 b, 3 c$, etc.).

With the exception of typical examples, bibliographic references are excluded from the profile descriptions in order to keep them succinct (the complete list of references is provided in Supplementary Materials).

The following aspects are described for each identified profile:

- A brief description of the impact of environmental degradation on migration;

- Mobility patterns-synthesis of the observed migration patterns;

- Policies-description of existing or absent policies;

- Geographic hotspot-list of places where the phenomena is acute;

- Typical example-description of an illustrative case study based on empirical research;

- Documents-number of scientific articles upon which the profile is based; 
- Confidence-we attempted to qualify each profile with the metrics of the IPCC (https: //www.ipcc.ch/site/assets/uploads/2017/08/AR5_Uncertainty_Guidance_Note.pdf, accessed on 24 September 2019). We estimated the degree of "agreement" (consensus between researchers/findings with the following qualifiers: High, medium, low) and "evidence" (number and extent of case studies with the following qualifiers: robust, medium, limited). The evaluation of evidence and agreement gives an indication of the level of confidence. If all case studies conclude, for example, that droughts lead to international migration (high agreement), but that these case studies are sparse (limited evidence), the level of confidence remains low. An explanatory note is provided for each qualifier.

\subsection{Profile 1: Mountainous Areas and Regional Migration}

Mountainous areas are particularly vulnerable to climate change. Considering only slow-onset degradation, this can take the form of temperature and rainfall variability, greater seasonal unpredictability, glacial melting, and serious losses of biodiversity.

Studies tend to show a correlation between unpredictable and changing weather patterns and the decision to migrate. Agriculture in mountainous areas is not sustainable year-round, and migration is often a means to counterbalance lost income during times of low production. The few existing papers on glacial melting, all based on ethnographic fieldwork, underline that while melting can hardly be considered a direct driver of migration, a sudden scarceness of water in the near future should not be underestimated. This situation could severely affect agricultural production in rural mountainous areas, as well as water availability for nearby urban areas.

- Mobility patterns: Migration from mountainous areas is described as mainly internal and oriented towards regional urban centers. Proximity to urban centers often defines the temporality of migration patterns. Those who live close to a city may commute frequently, whereas more remote populations often migrate for longer periods. Non-mobile people - often the elderly and women with children-are particularly vulnerable to climate change in mountainous areas.

- Policies: No specific mentions.

- Geographic hotspot: Andes, Himalayas, mountainous African areas.

- Typical example: Milan et al. (2014) [5] focused on three rural communities in the Central Highlands of Peru, where traditional rain-fed agriculture is the most important economic activity. This article highlights differences in livelihoods and human mobility patterns between households located at different altitudes. In the lowlands, one or more members of most households commute daily to the nearby city. In the highlands, households (or some members) often resettle there. In both cases, circular migration patterns (including daily mobility) can be identified as households combine scarce agricultural income with urban income, rather than abandoning their arable land.

- Documents: Approximately 15 articles.

- Confidence: Medium agreement (very heterogeneous contexts, but similar findings)/limited evidence (studies spread over different areas).

\subsection{Profile 2: Loss of Biodiversity and Land/Forest Degradation}

Land or forest degradation is usually described as a migration driver in combination with other types of environmental degradation (desertification, drought, rainfall variability, land availability, etc.). Tacoli (2011) [6] specifies that "in the context of gradual climate change, it is possible to identify precipitating events, such as unusually harsh droughts, as tipping points from which livelihoods need to change radically in order to ensure survival." Based on case studies in Burkina Faso and north-eastern Brazil, Neumann et al. (2015) [7] showed that land degradation can have a larger impact on migration decisions than water availability. Almost no empirical research describes the potential interrelations between biodiversity loss and migration. 
The conclusions from the literature related to loss of biodiversity and land degradation remain rather vague regarding mobility patterns. Overall, however, the processes identified are generally close to those associated with Profile 1.

- Mobility patterns: Migration patterns are internal. They are often described as temporary or seasonal, generally from rural areas towards urban centers.

- Policies: No specific mentions. Tacoli (2011) [6]—“Policies tend to veer between controlling migrants and ignoring them, with little in between."

- Geographic hotspot: All the continents are potentially exposed.

- Typical example: Mentioned in the text.

- Documents: Approximately 15 articles.

- Confidence: Low agreement (limited comparison due to heterogeneous contexts)/limited evidence (very few studies).

\subsection{Profile 3a: Sea Level Rise in Small Island Contexts without Relocation}

Small island states often consist entirely of low-lying atolls. Sea level rise, ocean acidification, and increases in extreme surface temperatures compromise the human security of a large number of people. With sea level rise projected to continue going forward, the physical integrity of low-lying islands is under threat. Due to intensifying storms, a gradual increase in soil salinity may cause these islands to become uninhabitable even before their complete submersion.

- Mobility patterns: Studies of small islands are dominated by qualitative approaches focused on the experiences and perceptions of the population. Researches usually aim to better understand migratory decisions. Studies highlight that even where climate change and environmental degradation have been highly politicized, the key objective of migration in atoll states remains improved access to employment opportunities and affiliated social and economic benefits, such as better housing, health care, and education services. This reveals the complexity of the environment-migration nexus in situations where the impacts of climate change are evident and irreversible.

- Policies: See "Island communities and relocation".

- Geographic hotspot: Small islands mainly in the Pacific but also Oceania. There is an abundance of case studies on Tuvalu and Kiribati.

- Typical example: Mortreux and Barnett's (2009) [8] study of Tuvalu shows that most migrants do not cite climate change as a primary reason to leave. People in Funafuti wish to remain where they are for reasons of lifestyle, culture, and identity. Concerns about the impacts of climate change are not currently a significant driver of migration from Funafuti, and do not appear to be a significant influence on those who intend to migrate in the future.

- Documents: Approximately 30 articles.

- Confidence: Medium agreement (complexity of decision-making leading to different behavior)/robust evidence (fair number of studies).

\subsection{Profile 3b: Island Communities and Relocation}

The resettlement (resettlement and relocation are used as synonyms) of at-risk populations is often described as the ultimate consequence of rising sea levels. Many island communities in the Pacific will have to relocate during the coming years. Most work in the area of climate-induced relocation concentrates on future resettlements, yet climate-related relocations are presently ongoing.

In some cases, relocation can happen within the island, but in other cases communities have limited options for domestic relocation, especially on atoll islands.

For some island nations, the risk of partial or complete submersion raises the question of statelessness. Countries such as the Maldives and Tuvalu may become uninhabitable due to submersion. Some islands have already disappeared (Holland Island, New Moore/South Talpatti) or led to relocation (Carteret Islands), but these territories are not sovereign nations and therefore do not lead to situations of statelessness. 
- Mobility patterns/Policies: The implementation of relocation policies is a characteristic feature of this profile. An extensive body of research exists on involuntary relocation caused by development projects, such as dam construction, mining activities, and natural hazards. Studies have shown that development-induced relocations expose affected communities to risks of impoverishment as a result of losses of livelihood, resources, and civil rights. Many development schemes demonstrate a lack of concern for the social dynamics of displaced communities. Some studies point out interesting measures, such as exchange programs between hosts and resettled communities in order to reduce the risk of conflict. However, relocation is still often described as a strategy of last resort, as any forced move may be maladaptive for those involved, and islanders often resist the idea due to place-based attachment and cultural issues that are of paramount importance.

- Some cases of bilateral migration arrangements at the regional level are described as possible solutions to address statelessness, along with various protective measures, but many authors consider their effective responses inadequate. Exploratory policies such as transnational ex-situ citizenship are also currently being discussed.

- Typical examples (Inland): Charan et al. (2017) [9] explored the interior resettlement of a village in Fiji, underlining obstacles such as disputes over land rights, social cohesion, and emotional and traditional attachments to place. The article's authors point out that relocation as a climate change adaptation strategy must be considered as a last resort particularly because of the high financial and social costs. Communities differ in levels of vulnerability, and a systematic preliminary assessment should be carried out to determine the most appropriate adaptation strategies.

- Typical examples (Outland): Edwards (2013) [10] examined the relocation of the Carteret Islands, Papua New Guinea. Resettlement had been on the agenda of local government for four decades, and several attempts were made to resettle islanders to Bougainville. All attempts were unsuccessful and resulted in the relocated population returning to the atoll. Findings highlight the issues faced by the displaced community and other stakeholders. Landlessness, unemployment, and support from host communities are key components in the success of resettlement schemes. Replicating island life was only possible on another small island.

- Documents: Approximately 30 articles.

- Confidence: High agreement (good knowledge of relocation issues)/robust evidence (fair number of studies).

\subsection{Profile 3c: Sea Level Rise in Coastal Areas without Policies}

Sea level rise will expose millions of people to increasing coastal hazards and potential land loss. Compared to cases where relevant policies are implemented, here the research tends to focus on the interaction between climate change impacts and migration. SLR can displace populations through coastal erosion, inundation cycles, and increasing soil salinity, with the latter having adverse effects on agricultural production.

- Mobility patterns: Migration processes tend to be regional, but cross-border migration is also documented. Mobility varies from temporary to permanent. Increasing environmental risks make planned or unplanned movements unavoidable in the near future.

- Policies: None (profile without policy).

- $\quad$ Typical example: Chen and Mueller (2018) [11] examined the extent to which farmers in coastal Bangladesh can adapt to increased sea/freshwater flooding and soil salinity. The latter is found to have direct effects on internal and international migration patterns. This research suggests that migration is driven, in part, by the adverse consequences of salinity for crop production.

- Geographic hotspots: Bangladesh is by far the most studied area. Other studies focus on Asia (i.e., Indonesia, Vietnam, India). Despite the fact that many coastal areas of China are densely populated, these remain relatively poorly studied in terms of migration. 
In Africa, studies are mainly located on the western coast, from Mauritania to Nigeria. Egypt is also documented. Latin America is mainly discussed in overviews at the global level, but some case studies document situations in central America (i.e., Belize). Oceania and Europe remain poorly documented.

- Documents: Approximately 70 articles.

- Confidence: Medium agreement (variety of migratory patterns)/robust evidence (significant number of studies).

\subsection{Profile 3d: Sea Level Rise in Coastal Areas with Relocation/Resettlement Programs}

Mobility patterns and policies: Within this profile, migratory or relocation (resettlement and relocation are used as synonyms) processes are internal, including towards cities. Relocation programs implemented by national authorities often result in experiences of social injustice and socio-economic impoverishment in the relocated population. To explain why most relocation policies fail, authors point to a lack of adaptive, community-based integration programs for migrants, and criticize the fact that governmental programs are often designed for emergency responses (i.e., to natural disasters) and do not address long-term or gradual displacement.

- Typical examples: Shishmaref, Alaska (US) is widely documented in discussions of relocation measures (i.e., Hamilton et al. 2016) [12]. In this region, relocation is the only strategy that can protect lives and infrastructure. However, the studies point out the ineffectiveness of existing migration and adaptation policies. There is currently no US policy that supports resettling "climate refugees". The applied policies are a patchwork of various government programs and procedures that are often insufficient. Saint-Louis (Senegal) is designated by UN-Habitat as the African city most threatened by sea level rise (i.e., Zickgraf 2016) [13]. Communities that rely on the fishing industry face dramatic coastal erosion. National authorities have already started to displace the population to inland camps. These camps are precarious and were intended only for temporary use.

- Geographic hotspots: Cf. Profile 3c.

- Documents: Approximately 20 articles.

- Confidence: High agreement (good knowledge of relocation issues)/robust evidence (identified areas are well documented).

\subsection{Profile 4a: Desertification in Sub-Saharan Africa: Seasonal Migration}

Sub-Saharan Africa is greatly affected by desertification (desertification is defined as a combination of demonstrated environmental degradations, including rain deficit, temperature increase, and soil degradation). In this environmental context, more frequent droughts can trigger migration. However, the climatic factor is only one of many fragile elements in this region, where access to mobility is anchored within structures of culture and tradition, particularly for pastoralist and nomadic populations. Environmental changes can modify pre-existing migration patterns. People who depend on natural resource industries for their livelihoods are the most vulnerable, since the agricultural sector is the largest employer. Migration can create land tenure tensions and conflicts in places of arrival.

Rainfall deficits and rising temperatures seem to be the two hazards that have the greatest impact on internal or inter-regional migration, mainly temporarily.

- Mobility patterns: The predominant flows are from rural areas to urban centers, although the literature notes occasional rural-to-rural migration. While young men are over-represented in rural out-migration, there is a growing trend toward a feminization of migration. Lower-educated people are more likely to respond to environmental shock through short-distance migration, whereas highly educated individuals may be candidates for more distant migrations.

- Policies: Few studies document concrete policies. Besides cooperative bilateral projects, a vast international project aims to fight desertification in Sahel and the Horn of Africa. The controversial "great green wall" involves planting a tree wall that crosses 
11 countries between Dakar and Djibouti. Its goals are to slow desertification and provide new economic opportunities for climatic resilience.

- While some policy recommendations in the literature address the environment directly, others focus on migration. Mobility is poorly perceived, although migration is an integral part of sub-Saharan African culture. Regarding the environment, recommendations to prevent and reduce the risks related to rainfall deficits are predominant. For example, suggestions include the establishing of small-scale irrigation systems, distributing heat- and drought-resistant crops, and improving access to local weather forecasts in order to prevent trapped populations. In terms of migration, authors underline that it diversifies incomes in semi-arid regions and is therefore a means of adapting to droughts. They suggest that national policies and development policies must take into account this characteristic of Sahelian populations. Finally, as most migration flows towards urban centers, the relevant authorities are encouraged to implement urbanization policies in line with this phenomenon. Moreover, policy proposals need to reconcile economic growth and development goals with pastoralism, agriculture, and climate change adaptation measures.

- Geographic hotspots: Burkina-Faso, Mali, Niger, Senegal, Chad, Benin, Ghana, Nigeria, Burundi, Ethiopia, Kenya, Somalia, Sudan, Tanzania, Uganda.

- Typical example: Rademacher-Schulz and al. (2013) [14] examined the interrelationships between rainfall variability, livelihood/food security, and migration in Northern Ghana. This research addresses dry-season migration, and whether this migration pattern is a coping or adaptation mechanism. Using a mixed-methods approach, the results show that a common household strategy is dry-season domestic migration to more suitable farming areas and mining sites.

- Documents: About 70 articles.

- Confidence: High agreement (heterogeneous contexts but general migratory patterns can be distinguished)/medium evidence (high number of studies but great variety of methods and scales of analysis).

\subsection{Profile 4b: Desertification in Sub-Saharan Africa: International Migration}

- Mobility patterns: Whereas desertification often leads to internal migration, it can also disrupt international migration due to reduced financial resources (poverty trap). $\mathrm{Ob}$ servations show that environmental changes tend to slow out-migration, especially in regions that are highly dependent on natural resources. Nevertheless, environmental changes may influence other migration drivers (i.e., conflicts over natural resources), which in turn may favor international migration.

- Typical example: Henry et al. (2004) [15] suggested that people from the drier regions of Burkina Faso are more likely than those from wetter areas to engage in both temporary and permanent migration to other rural areas. Short-term rainfall deficits also tend to increase the risk of long-term migration to rural areas and decrease the risk of shortterm moves to distant destinations. On the other hand, Cai and al. (2016) showed a significant relationship between temperature and international out-migration in agriculture-dependent African countries.

- Policies: As stated in Profile 4a, policies are only marginally taken into account in case studies focused on Africa. Policy recommendations underline the need to develop mechanisms that maximize the benefits of remittances.

- Geographic hotspots: Cf. Profile 4a

- Documents: Approximately 30 articles

- Confidence: Medium agreement (debated results, but tendentially more agreement upon a weak relationship between environmental degradation and international migration)/medium evidence (high number of studies but great variety of methods and scales of analysis). 


\subsection{Profile 4c: Desertification in Central America}

Migration from Mexico to the US is a dominant case in the literature on desertification and migration in Central America. Many authors have shown a correlation between migration from rural (and often marginalized) regions and lower rainfall amounts and/or lower crop yields. Higher levels of rainfall reduce migration during the following years. However, some studies have shown contrasting results. This might be explained by the fact that the Mexican states under study record different rainfall averages (i.e., drylands, the Central-Western region). Furthermore, in some cases, a decline in rainfall may reduce household capital and thereby inhibit long-distance international migration. Only in places with high prior emigration rates and strong Mexico-US social networks was declining rainfall associated with migration to the US. International migration is thus a more common adaptation strategy to climate variability in particularly harsh times or extended droughts, and from places with well-established migrant networks. Mexico-US migration flows are sensitive to climate and environmental change only under specific climatic and social conditions.

Besides Mexico, some studies have shown that droughts augment migration in Guatemala, Haiti, northern Latin America, and the Caribbean.

- Mobility patterns: Rural to urban migration within Mexico; international migration from Mexico, Guatemala, Haiti, and other central American countries to the US.

- Typical examples: Nawrotzki, Riosmena and Hunter (2013) [16] found that a decrease in precipitation is significantly associated with US-bound migration, but only for dry Mexican states. However, the same authors showed in another article that declining precipitation relative to the average may reduce emigration in areas undergoing rainfall deficits.

- Policies: Policies are mostly not discussed. Some studies examine programs in the Mexican state of Chiapas where the government has provided financial assistance to residents in order to reduce out-migration.

- Geographic hotspots: Mexico, Nicaragua, Guatemala, Costa Rica, Haiti, the Dominican Republic, and the US (as destination).

- Documents: Approximately 30 articles.

- Confidence: Low agreement (debated results)/robust evidence (well-documented case).

\subsection{Profile 4d: Desertification in the US and the "Dust Bowl"}

The Dust Bowl of the 1930s is often taken as an example of mass exodus following drought and desertification. Authors use this case as a historical analogue to today's displacements following slow-onset events. The famous 1939 John Steinbeck novel The Grapes of Wrath—as well as John Ford's celebrated Hollywood film version (1940)—are illustrative of the "Dust Bowl Migration". This migration is viewed through a multifaceted perspective, resulting from a combination of economic (recession, falling commodity prices) and environmental factors (droughts and storms). Most migrants were young, married couples with children who left rural Oklahoma and neighboring states for southern California.

Concerning today's environmental migration patterns in the US, studies almost exclusively address sudden-onset disasters (Hurricane Katrina in 2005 being an emblematic case).

- Mobility patterns: Internal migrations from the Great Plains (Arkansas, Kansas, Oklahoma, North Texas, and parts of Colorado and Missouri) to the western US (Pacific Coast) or from rural to urban areas within the Great Plains.

- Typical example: McLeman and Hunter (2010) [17] used Dust Bowl migration as an interesting analogue to help identify general, causal, temporal, and spatial dimensions of today's climate migrations.

- Policies: This case offers an interesting historical perspective on policies addressing environmental displacement. Authors note that the governmental policies and programs that have proven most beneficial to drought-stricken farmers were actually designed to stabilize commodity prices, provide income stability for farmers, and 
create job opportunities for the unemployed. Other federal programs assisted drought migrants. For example, worker camps in southern California were managed by the federal Farm Security Administration in order to provide safe temporary housing for migrants. The authors note that this effort counteracted the attempts of some state and county governments in California to actively discourage migrants and obstruct their access to publicly funded services.

- Geographic hotspot: The Great Plains of the western US.

- Documents: Approximately 10 articles.

- Confidence: High agreement (consistent results)/robust evidence (low amount of research but focused on the same area).

\subsection{Profile 4e: Desertification in the Rest of the World}

In addition to the regions identified in the previous profiles, desertification is acute in various places around the world. However, it is difficult to describe a global trend for these cases because their research interests and geographical contexts are too heterogeneous. Considering that they add value to our understanding of desertification impacts, these cases are briefly described in the following list:

- Austral Africa (6 articles): Some case studies describe how villagers cope with droughts. Support from local leaders and chiefs has been replaced by state support. Long-term migration is no longer popular, with many people preferring to wait for food aid. Other studies give historical perspectives of migratory patterns across this part of the continent. Confidence: Medium agreement (limited findings)/limited evidence (few studies).

- Middle East (6 articles): While climate change is not currently the main driver of migration flows, it does appear to contribute substantially, in that worsening climatic conditions are likely to exacerbate future migration flows. This region is affected by desertification processes, which contribute to migratory systems characterized by rapid and extensive rural-urban migration, established and ongoing cross-border and internal displacement due to war and conflicts, and emigration to neighboring oil-rich countries, as well as Europe, North America, and Australia. Confidence: Low agreement (limited findings)/limited evidence (few studies).

- European countries (4 articles): Although the South of Europe is affected by desertification, few studies underline the migratory impacts, tending to either emphasize the complexities of the relationships between these processes or to observe no relationship at all. Europe is also discussed as a potential destination area. There is a tendency in media discourses to consider that climate change will lead to an increased number of migrants seeking asylum in Europe. Confidence: Low agreement (limited findings)/limited evidence (few studies).

- China (12 articles): Even though a number of articles focus on China, they tend to approach migration through a historical perspective over the last few centuries. The few case studies about the current situation discuss the role of environmental factors in migration decisions, but mobility patterns are poorly documented. Policies are not discussed. Confidence: Low agreement (limited findings)/limited evidence (fair number of studies, but migration is under-documented; political factors).

- South America (24 articles): Desertification processes on this continent are often related to the ENSO oscillations. Droughts in Brazil are particularly well documented and have led to massive decreases in agricultural production and an increase in internal migration, mainly urbanization. Confidence: High agreement (consistent findings)/medium evidence (fair number of studies focused on Brazil, but other areas are poorly documented).

\subsection{Profile 5: Slow-Onset Processes and Immobilities}

The literature on immobility following environmental hazards or forecasted degradation is recent and growing. Research on environmental immobilities clearly distinguishes 
involuntary immobility ("trapped populations") and voluntary immobility (people who choose not to migrate for cultural, social, or psychological reasons). Immobilities resulting from slow-onset events have not been very well explored in the literature, but the following examples outline the issue.

- Policies: Few specific mentions are made. Various studies underline that-especially in the case of relocation-local populations often resist policies that do not take into account their wishes and needs regarding future livelihood conditions.

- Typical examples (Involuntary immobility): Slow-onset events may prevent migration. As rainfall averages increased in the Western Highlands of Guatemala, rural mountain communities suffered from a decrease in local diversification options. Declining outmigration meant fewer remittances, and therefore further local income reductions. These trends expose populations to becoming trapped in places that are extremely vulnerable to climate change. In other regions, droughts or reduced rainfall might reduce migration flows when dwindling resources are allocated to basic needs rather than to out-migration. The remaining receivers of remittances are also immobile populations who may be particularly vulnerable to the effects of climate variability (i.e., Gray and Wise 2016; Milan and Ruano 2014; Van der Geest 2011). [18-20].

- Typical examples (Voluntary immobility): Despite the adverse effects of climate change, in some contexts, people may decide to stay for socio-psychological reasons (i.e., place attachment and place identity concepts). Studies have shown the importance of attachment in an Australian coastal community threatened by sea level rise, and others have shed light on people's decision to stay in Tuvalu despite growing SLR risks. They are greatly attached to their ancestral lands and wish to adapt in situ (i.e., Adams and Kay 2019; O'Neill and Graham 2016; Mortreux and Barnett 2009) [8,21,22].

- Geographic hotspots: Cases studies documenting these situations are mainly within the contexts of islands and mountainous areas.

- Documents: Approximately 10 articles.

- Confidence: Low agreement (heterogeneous findings)/limited evidence (few studies).

\section{Conclusions}

This paper has attempted to transcend hot-spot identification/vulnerability assessment exercises by producing profiles that simultaneously take into account the environment, mobility patterns, and-where possible-relevant policies. Each profile is categorized according to the level of confidence associated with the related research results (Figure 2). This allows us to spot research consensus and research gaps.

\begin{tabular}{|r|l|l|}
\hline 1 & Mountainous areas and regional migration & Medium agreement / Limited evidence \\
\hline 2 & Loss of biodiversity and land/forest degradation & Low agreement / Limited evidence \\
\hline 3a & Sea level rise in small island contexts without relocation & Medium agreement / Robust evidence \\
\hline 3b & Island communities and relocation & High agreement / Robust evidence \\
\hline 3c & Sea level rise in coastal areas without policies & Medium agreement / Robust evidence \\
\hline 3d & Sea level rise in coastal areas with relocation/resettlement programmes & High agreement / Robust evidence \\
\hline 4a & Desertification in sub-Saharan Africa : seasonal migration & High agreement / Medium evidence \\
\hline 4b & Desertification in sub-Saharan Africa : international migration & Medium agreement / Medium evidence \\
\hline 4c & Desertification in Central America & Low agreement / Robust evidence \\
\hline 4d & Desertification in the US and the "Dust Bowl" & High agreement / Robust evidence \\
\hline 4e & Desertification in the rest of the world & Agreement and evidence vary between regions \\
\hline 5 & Slow-onset processes and immobilities & Low agreement / Limited evidence \\
\hline
\end{tabular}

Figure 2. Identified profiles with IPCC-style levels of confidence.

This approach was exploratory and did not always prove successful. Some profiles remain poorly documented in the literature or are too heterogeneous to be really useful (i.e., Profiles $4 \mathrm{e}$ and 5). Profiles $1,3 \mathrm{a}-3 \mathrm{~d}$ and $4 \mathrm{a}-4 \mathrm{~d}$, however, allow us to identify interesting features of the processes at stake. 
It is worth noting that there is still a strong imbalance in geographical distribution in the literature, which also appears in the identified profiles. In the field of climate change and migration research, areas known as hotspots-such as Shishmaref, Tuvalu-Kiribati, or Bangladesh - catch the attention of many researchers, while other densely populated regions affected by the impacts of climate change remain under-studied, such as northeastern Africa, the Nile basin, coastal African areas, South America, China, and others. Research on the Global North is scarce, and tends to focus on remote regions connected to Indigenous cultures, as in Shishmaref. This observation aligns with a general research bias towards "exotic" cases studies, as described by Piguet et al. (2018) [3].

Certain similarities that transcend the particularities of the identified profiles can be shown. Slow-onset environmental stress is usually framed as being part of and interacting with the multiple drivers of migration (multicausality). Even in the evident case of sea level rise, other factors interact to shape mobilities. Throughout most profiles, research indeed shows that only certain individuals in a given community engage in migratory flows, while others remain. Therefore, remittances may play an important role as a means of income for those who stay in areas where regular employment is not available due to climatic stresses.

Finally, it appears that several important topics are overlooked in the empirical literature on slow-onset environmental degradation. First, few studies address existing policies. In general, migration-related policies are only superficially discussed in the recommendations chapters of this literature. It is likely that these (i.e., relocation or resettlement policies) are more often studied in the context of rapid-onset environmental degradations. Second, destination areas are only marginally considered. For example, some research underlines the importance of urbanized areas as destinations, but does not go further into considering the contextuality of these processes and their consequences.

On the basis of these findings, we suggest that additional analyses of the existing literature, as well as new empirical research, are needed. The profiles identified in this paper will hopefully help to target the neglected fields of inquiry for future research, and lead to more precise analyses and comparisons of displacements. We hope they will also pave the way for evidence-based policies that might mitigate the progressive but threatening consequences of environmental change.

Supplementary Materials: The following are available online at https://www.mdpi.com/article/10 $.3390 /$ su131810235/s1.

Author Contributions: Both authors contributed equally to this paper, All authors have read and agreed to the published version of the manuscript.

Funding: This research was partially funded the Internal Displacement Monitoring Centre (IDMC). Institutional Review Board Statement: Not applicable.

Informed Consent Statement: Not applicable.

Data Availability Statement: No new data were created or analyzed in this study. Data sharing is not applicable to this article.

Conflicts of Interest: The authors declare no conflict of interest.

\section{References}

1. United Nations Framework Convention on Climate Change. Slow Onset Events_Technical Paper; UNFCCC: Bonn, Germany, 2012.

2. Cattaneo, C.; Beine, M.; Fröhlich, C.; Kniveton, D.; Martinez-Zarzoso, I.; Mastrorillo, M.; Millock, K.; Piguet, E.; Schraven, B. Human Migration in the Era of Climate Change. Rev. Environ. Econ. Policy 2019, 13, 189-206. [CrossRef]

3. Piguet, E.; Kaenzig, R.; Guélat, J. The uneven geography of research on "environmental migration". Popul. Environ. 2018, 39, 357-383. [CrossRef]

4. IDMC. No Matter of Choice: Displacement in a Changing Climate-Research Agenda and Call for Partners; Internal Displacement Monitoring Centre: Geneva, Switzerland, 2018; Available online: https://www.internal-displacement.org/publications/nomatter-of-choice-displacement-in-a-changing-climate (accessed on 24 September 2019). 
5. Milan, A.; Ho, R. Livelihood and migration patterns at different altitudes in the Central Highlands of Peru. Clim. Dev. 2013, 6, 69-76. [CrossRef]

6. Tacoli, C. Not Only Climate Change: Mobility, Vulnerability and Socio-Economic Transformations in Environmentally Fragile Areas of Bolivia, Senegal and Tanzania. IIED Human Settlements Working Paper; International Institute for Environment and Development: London, UK, 2011; Available online: https://pubs.iied.org/sites/default/files/pdfs/migrate/10590IIED.pdf (accessed on 24 September 2019).

7. Neumann, K.; Sietz, D.; Hilderink, H.; Janssen, P.; Kok, M.; van Dijk, H. Environmental drivers of human migration in drylands-A spatial picture. Appl. Geogr. 2015, 56, 116-126. [CrossRef]

8. Mortreux, C.; Barnett, J. Climate change, migration and adaptation in Funafuti, Tuvalu. Glob. Environ. Chang. 2009, 19, 105-112. [CrossRef]

9. Charan, D.; Kaur, M.; Singh, P. Customary Land and Climate Change Induced Relocation-A Case Study of Vunidogoloa Village, Vanua Levu, Fiji. In Climate Change Adaptation in Pacific Countries; Springer International Publishing: Berlin/Heidelberg, Germany, 2017; pp. 19-33.

10. Edwards, J.B. The Logistics of Climate-Induced Resettlement: Lessons from the Carteret Islands, Papua New Guinea. Refug. Surv. Q. 2013, 32, 52-78. [CrossRef]

11. Chen, J.; Mueller, V. Coastal climate change, soil salinity and human migration in Bangladesh. Nat. Clim. Chang. 2018, 8, 981-985. [CrossRef]

12. Hamilton, L.C.; Saito, K.; Loring, P.A.; Lammers, R.B.; Huntington, H.P. Climigration? Population and climate change in Arctic Alaska. Popul. Environ. 2016, 38, 115-133. [CrossRef] [PubMed]

13. Zickgraf, C.; Vigil Diaz Telenti, S.; de Longueville, F.; Ozer, P.; Gemenne, F. The Impact of Vulnerability and Resilience to Environmental Changes on Mobility Patterns in West Africa; World Bankd: Washington DC, USA, 2016.

14. Rademacher-Schulz, C.; Schraven, B.; Mahama, E.S. Time matters: Shifting seasonal migration in Northern Ghana in response to rainfall variability and food insecurity. Clim. Dev. 2014, 6, 46-52. [CrossRef]

15. Henry, S.; Schoumaker, B.; Beauchemin, C. The Impact of Rainfall on the First Out-Migration: A Multi-level Event-History Analysis in Burkina Faso. Popul. Environ. 2003, 25, 423-460. [CrossRef]

16. Nawrotzki, R.J.; Riosmena, F.; Hunter, L. Do Rainfall Deficits Predict U.S.-Bound Migration from Rural Mexico? Evidence from the Mexican Census. Popul. Res. Policy Rev. 2013, 32, 129-158. [CrossRef] [PubMed]

17. McLeman, R.A.; Hunter, L. Migration in the context of vulnerability and adaptation to climate change: Insights from analogues. Wiley Interdiscip. Rev. Clim. Chang. 2010, 1, 450-461. [CrossRef] [PubMed]

18. Gray, C.; Wise, E. Country-specific effects of climate variability on human migration. Clim. Chang. 2016, 135, 555-568. [CrossRef] [PubMed]

19. Milan, A.; Ruano, S. Rainfall variability, food insecurity and migration in Cabricán, Guatemala. Clim. Dev. 2013, 6, 61-68. [CrossRef]

20. Van Der Geest, K. North-South Migration in Ghana: What Role for the Environment? Int. Migr. 2011, 49, e69-e94. [CrossRef]

21. Adams, H.; Kay, S. Migration as a human affair: Integrating individual stress thresholds into quantitative models of climate migration. Environ. Sci. Policy 2019, 93, 129-138. [CrossRef]

22. O'Neill, S.J.; Graham, S. (En)visioning place-based adaptation to sea-level rise. Geo Geogr. Environ. 2016, 3, e00028. [CrossRef] 